\title{
Greater Real-Life Diagnostic Efficacy of Allergen Molecule-Based Diagnosis for Prescription of Immunotherapy in an Area with Multiple Pollen Exposure
}

\author{
Ulbosin Saltabayeva ${ }^{a, b}$ Victoria Garib ${ }^{a, c}$ Marina Morenko ${ }^{a, b}$ \\ Rafail Rosenson $^{b}$ Zhanat Ispayeva $^{a}{ }^{d}$ Madina Gatauova ${ }^{b}$ Loreta Zulus $^{a}$ \\ Alexander Karaulov ${ }^{\mathrm{e}}$ Felix Gastager ${ }^{\mathrm{a}, \mathrm{f}}$ Rudolf Valenta ${ }^{\mathrm{a}, \mathrm{c}}$ \\ ${ }^{a}$ International Network of Universities for Molecular Allergololgy and Immunology, Vienna, Austria; ${ }^{b}$ Astana Medical \\ University, Astana, Kazakhstan; ' Division of Immunopathology, Department of Pathophysiology and Allergy \\ Research, Medical University of Vienna, Vienna, Austria; ${ }^{d}$ S.D. Asfendijarov Kazakh National Medical University, \\ Almaty, Kazakstan; ${ }^{e}$ Department of Clinical Immunology and Allergy, Sechenov First Moscow State Medical

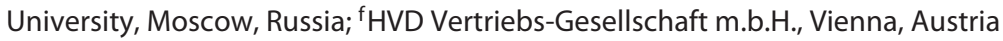

\section{Keywords}

Allergy · Allergen · Diagnosis · Component-resolved diagnosis · Skin prick testing · Molecular allergology Allergen-specific immunotherapy · Immunotherapy prescription · Economic efficacy

\begin{abstract}
Background: Allergen molecule-based diagnosis has been suggested to facilitate the identification of disease-causing allergen sources and the prescription of allergen-specific immunotherapy (AIT). The aim of the current study was to compare allergen molecule-based IgE serology with allergen extract-based skin testing for the identification of the diseasecausing allergen sources. The study was conducted in an area where patients are exposed to pollen from multiple sources (trees, grasses, and weeds) at the same time to compare the diagnostic efficiency of the 2 forms of diagnosis. Methods: Patients from Astana, Kazakhstan, who suffered from pollen-induced allergy $(n=95)$ were subjected to skin
\end{abstract}

U. Saltabayeva and V. Garib are both first authors.

\section{KARGER}

E-Mail karger@karger.com www.karger.com/iaa
C 2017 The Author(s)

Published by S. Karger AG, Basel

Karger

Open access

This article is licensed under the Creative Commons Attribution NonCommercial-NoDerivatives 4.0 International License (CC BYNC-ND) (http://www.karger.com/Services/OpenAccessLicense). Usage and distribution for commercial purposes as well as any distribution of modified material requires written permission. prick testing (SPT) with a local panel of tree pollen, grass pollen, and weed pollen allergen extracts and IgE antibodies specific for marker allergen molecules (nArt $\vee 1, n$ Art $\vee 3$, $\mathrm{rAmb}$ a $1, \mathrm{rPhl} \mathrm{p} 1, \mathrm{rPhl} \mathrm{p} 5$, rBet $v 1$ ) were measured by $\mathrm{lm}$ munoCAP. Direct and indirect costs for diagnosis based on SPT and marker allergen-based IgE serology as well as direct costs for immunotherapy depending on SPT and serological test results were calculated. Results: The costs for SPT-based diagnosis per patient were lower than the costs for allergen molecule-based IgE serology. However, allergen moleculebased serology was more precise in detecting the diseasecausing allergen sources. A lower number of immunotherapy treatments $(n=119)$ was needed according to molecular diagnosis as compared to extract-based diagnosis $(n=275)$, which considerably reduced the total costs for diagnosis and for a 3-year treatment from EUR 1,112.30 to 521.77 per patient. Conclusions: The results from this real-life study show that SPT is less expensive than allergen molecule-based diagnostic testing, but molecular diagnosis allowed more precise prescription of immunotherapy which substantially reduced treatment costs and combined costs for diagnosis and treatment.

(c) 2017 The Author(s) Published by S. Karger AG, Basel

Correspondence to: Dr. Victoria Garib

Department of Pathophysiology and Allergy Research, Medical University of Vienna Währinger Gürtel 18-20

AT-1090 Vienna (Austria)

E-Mail viktoryia.garib@meduniwien.ac.at 


\section{Introduction}

More than 25 years ago the first study was published which showed that recombinant allergens can be used for diagnosis of allergic sensitization based on IgE serology using birch pollen allergy as a model [1]. Over time, the number of recombinant allergens from various allergen sources increased and recombinant allergen-based in vitro diagnostics became available in the form of component-resolved diagnostics in the ImmunoCAP test system, which allowed sensitization profiles of allergic patients to be established [2]. Allergen molecule-based diagnosis continued to develop and the first multiplex allergen test based on microarrayed allergens was described in 2002 [3]. A major advantage of recombinant allergen-based diagnosis over allergen extract-based diagnosis is the possibility that allergen-based tests allow to discriminate cosensitization from cross-sensitization $[4,5]$.

It was proposed that allergen-molecule-based testing may be especially useful for a more accurate identification of the culprit allergen sources and thus may facilitate a more precise prescription of allergen-specific immunotherapy (AIT) $[6,7]$. This concept has gained support by studies in which a post hoc analysis of the outcome of AIT using allergen molecule-based diagnosis was performed $[8,9]$. Furthermore, several studies showed that clinicians would have changed the prescription of allergen extracts for AIT substantially based on allergen-molecule-based diagnosis $[10,11]$.

AIT is the only disease-modifying treatment for allergy with long-lasting effects and one of the major advantages is that it uses the immune system for defending the allergic patient against symptoms (immunological mechanisms of AIT) [12-14]. Another major advantage is that AIT is more cost-effective than pharmacotherapy [15]. However, AIT requires multiple treatments over several years and it is therefore important to identify the relevant allergen extracts precisely to save additional costs and unnecessary treatments. Here we conducted a real-life study in an area where patients are exposed simultaneously to several different pollen allergen sources. In Astana, Kazakhstan, the summer period is very short and characterized with an overlap regarding the pollination of trees, grasses, and weeds [16]. Furthermore, the area is flat and extremely windy with far distribution of pollen from different regions. These peculiarities make the determination of the sensitizing pollen allergen source very difficult in this region. The aim of the study was to compare the cost-efficacy of traditional allergen extract-based skin test diagnosis with IgE-serology based on purified marker allergens representing the common allergen sources for prescription of AIT.

\section{Subjects and Methods}

Patients ( $n=95$; 48 females, 47 males; age from 5 to 56 years, mean age 24 years) with seasonal allergic rhinitis referred to the Allergy and Respiratory Outpatient Unit of Astana Medical University were investigated. None of the patients had symptoms of asthma, $77.9 \%$ had mild to moderate rhinitis and $22.1 \%$ had severe rhinitis, $81.1 \%$ had sneezing, and $18.9 \%$ had obstruction. Ninetyfive percent of the patients suffered also from conjunctivitis. A family history of atopy was recorded for $86.3 \%$ of the patients.

The study was approved by the ethical committee of JSC Medical University of Astana and performed in accordance with the 7 th revision of the Declaration of Helsinki (2013). The written consent of the patients was obtained.

Patients filled out a questionnaire adapted and translated from the ISAAC format for local use. Further diagnosis of pollen allergy was based on a detailed medical history indicative of seasonal pollen allergy, a physical examination with emphasis on the upper respiratory tract, skin prick testing (SPT) with a panel of pollen allergen extracts, total IgE measurement, and determination of eosinophil cationic protein in serum. The analysis of nasal smears for the presence of eosinophils investigation, fiber optic nasal endoscopy, and/or rhinomanometry was performed in patients suspected of having polyposis. The 95 patients in our study were identified in a much larger group of patients with sensitization also to other allergen sources. However, only patients for whom pollen allergy was the major clinically relevant allergy were included in our study because only in such patients is pollen-specific AIT indicated.

The SPT standard panel contained pollen allergen extracts of local relevant pollen sources including a weed mix (Artemisia absinthium, Artemisia vulgaris, Solidago canadensis), grass mix (Arrhenatherum elatius, Dactylis glomerata, Festuca sp., Lolium sp., Phleum pratense, Secale cereale), and a tree mix (Alnus glutinosa, Betula pendula, Carpinus betulus, Corylus avellana), as well as with pollen extracts from mugwort pollen, Timothy grass, bluegrass, fescue, and birch (Burly, National Center of Hygiene and Epidemiology of the Ministry of Health; www.burly.kz/index.php?page= home-3). Pollen allergen extracts were prepared from locally collected pollen by extraction using a phosphate buffer and $0.9 \%$ sodium chloride. For storage, $4 \%$ phenol solution was added. Sodium chloride and histamine solution were used as negative and positive controls, respectively (Burly). The SPT results were read after 15 min according to European guidelines [17].

Allergen-specific IgE antibody levels were measured by ImmunoCAP (Thermo Scientific, Uppsala, Sweden) according to the manufacturer's recommendations. Six single allergen components were tested: nArt v 1, rArt v 3, rAmb a 1 (weed pollen allergens), rPhl p 1, rPhl p 5 (grass pollen), and rBet v 1 (birch pollen). sIgE levels $>0.35 \mathrm{kUA} / \mathrm{L}$ were considered positive.

The costs of in vivo testing included costs for allergen extracts, control solutions, materials, and physicians according to standardized local prices. Costs for in vitro testing comprised the costs for the tests, blood collection, and laboratory technician contribution.
94

Int Arch Allergy Immunol 2017;173:93-98 DOI: $10.1159 / 000477442$
Saltabayeva et al. 
Fig. 1. Frequencies of sensitization to tree pollen, grass pollen, and weed pollen as determined by skin prick testing with mixed allergen extracts (a), allergen extracts from single allergen sources (b), or recombinant marker allergens (c) in patients with seasonal allergic rhinitis $(n=95)$.

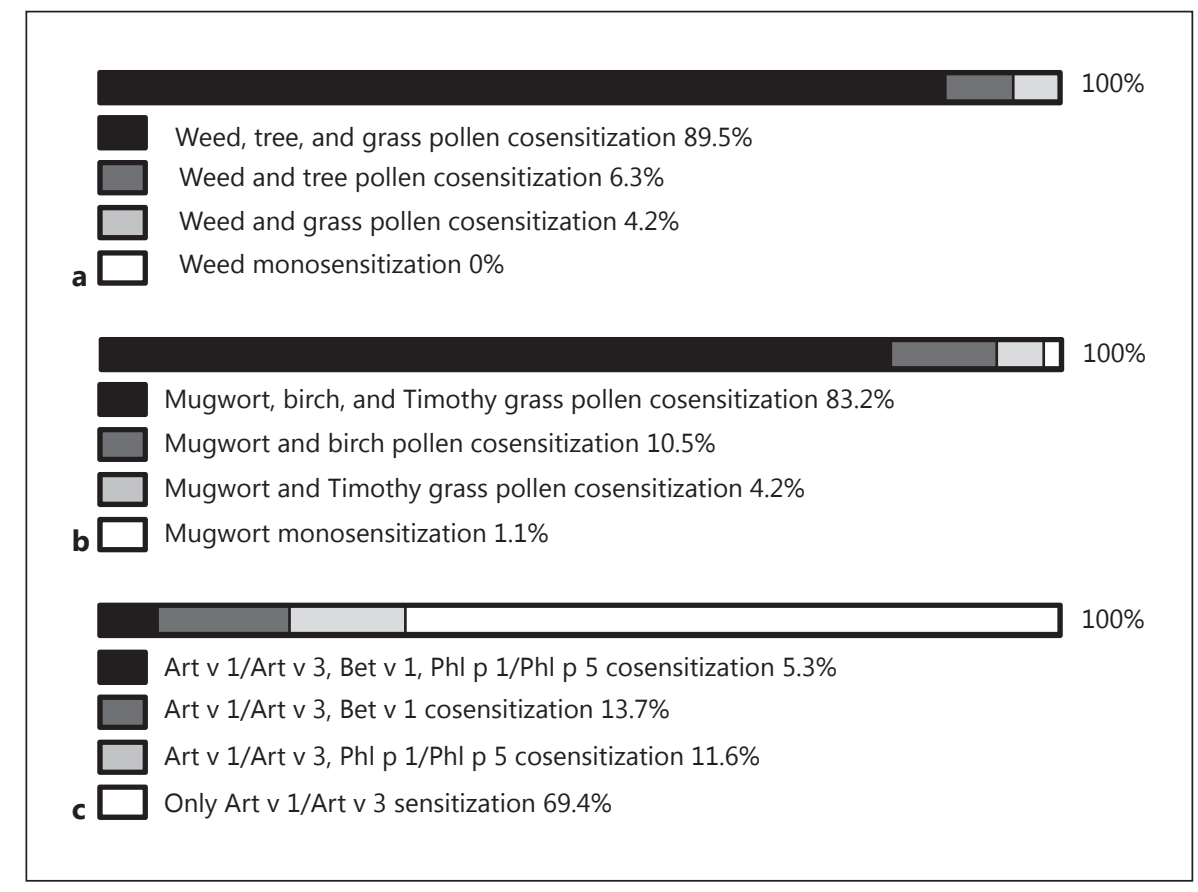

AIT costs included only the costs for the vaccine, but not the time spent by the patients and not the physicians' costs. The following pollen mixtures for AIT were available for treatment (Sevapharma, Prague, Czech Republic) for patients. A general grass pollen mixture included Agropyrum repens, Alopecurus pratensis, Anthoxanthum odoratum, Apera spica-venti, Arrhenatherum elatius, Avenastrum sp., Bromus erectus, Dactylis glomerata, Deschampsia flexuosa, Festuca sp., Holcus lanatus, Lolium sp., Phleum pratense, Poa pratensis, Secale cereal, and Trisetum flavescens. A tree pollen mixture consisted of Alnus glutinosa, Betula pendula, Carpinus betulus, and Corylus avellana. Patients with weed pollen allergy were treated by an "autumn pollen mixture," including Artemisia absinthium, Artemisia vulgaris, and Solidago canadensis. There was also the possibility for AIT by "pollen of medical herbs," including Ambrosia artemisiifolia, Artemisia absinthium, Artemisia vulgaris, Aster sp., Brassica napus, Chenopodium album, Chrysantheum hort., Chrysantheum leucant., Helianthus annuus, Iva xanthiifolia, Plantago lanceolata, Primula sp., Rumex acetosa, Solidago canadensis, Taraxacum officinale, and Urtica dioica. The AIT costs were calculated for 1 and 3 years of treatment, respectively.

Distribution of IgE sensitizations to allergen molecules, correlation rate, and economical efficacy were analyzed using IBM SPSS 20 and Microsoft Excel.

\section{Results}

Skin Testing Is Less Precise than Allergen

Molecule-Based Testing for the Identification of the

Culprit Allergen Sources

According to SPT with weed, tree, and grass pollen allergen extract mixes and single mugwort, birch, and Tim- othy grass pollen extracts, comparable results were obtained (Fig. 1a, b). More than $89 \%$ of the patients showed positive SPT reactions to weed, tree, and grass pollen mixes and therefore appeared to be cosensitized to 3 different allergen sources, $6 \%$ showed a weed and tree pollen cosensitization, and $4.2 \%$ a weed and grass pollen cosensitization (Fig. 1a). Similar results were observed for SPT with single allergen extracts from mugwort, birch, and Timothy grass pollen (Fig. 1b). More than $83 \%$ appeared triple-sensitized, $10.5 \%$ reacted to mugwort and birch pollen, and $4.2 \%$ to mugwort and Timothy grass pollen. One patient appeared monosensitized to mugwort.

When genuine marker allergens from mugwort (i.e., Art v 1, Art v 3), Timothy grass (Phl p 1, Phl p 5), and birch pollen (Bet v 1) were used for IgE-based serology, different results were obtained. Only $5.3 \%$ of the patients turned out to be genuinely cosensitized to mugwort, Timothy grass, and birch; $13.7 \%$ were cosensitized to mugwort and birch; and $11.7 \%$ had a cosensitization to mugwort and Timothy grass (Fig. 1c). The majority of patients (i.e., 69.4\%) were monosensitized to mugwort pollen as demonstrated by exclusive IgE reactivity to Art v 1 and/ or Art v 3.

It thus turned out that SPT and allergen moleculebased testing led to a fundamentally different choice of extracts for AIT. According to SPT results, 85 patients (i.e., $89.5 \%$ ) would have received AIT with 3 different allergen extracts covering weed, tree, and grass pollen
Economic Efficacy of Component-

Resoloved Diagnosis for AIT
Int Arch Allergy Immunol 2017;173:93-98 DOI: $10.1159 / 000477442$ 
Table 1. Economic efficacy of componentresolved diagnosis based on allergen molecules versus SPT for AIT treatment for patients with pollen-induced allergic rhinitis $(n=95)$

\begin{tabular}{|c|c|c|c|}
\hline & $\begin{array}{l}\text { Skin prick } \\
\text { testing }\end{array}$ & ImmunoCAP & $\begin{array}{l}\text { Economic } \\
\text { efficacy }\end{array}$ \\
\hline \multicolumn{4}{|l|}{ Diagnostic cost, EUR } \\
\hline Per patient & 23.4 & 92.20 & -68.80 \\
\hline For the group & $2,223.0$ & 8,759 & $-6,536.00$ \\
\hline \multicolumn{4}{|l|}{ AIT prescription, $n(\%)$} \\
\hline 1 extract & $0(0)$ & $66(69.4)$ & \\
\hline 2 extracts & $10(10.5)$ & $24(25.3)$ & \\
\hline 3 extracts & $85(89.5)$ & $5(5.3)$ & \\
\hline Mean extracts per patient & 2.9 & 1.3 & \\
\hline \multicolumn{4}{|l|}{ AIT cost, EUR } \\
\hline Per year per patient & 370.76 & 173.92 & 196.84 \\
\hline Per 3 years per patient & $1,112.30$ & 521.77 & 590.53 \\
\hline \multicolumn{4}{|c|}{ Total cost (diagnostic/AIT), EUR } \\
\hline Per patient & $1,135.70$ & 613.97 & 521.73 \\
\hline For the group & $107,891.50$ & $58,327.15$ & $49,564.35$ \\
\hline
\end{tabular}

SPT, skin prick testing; AIT, allergen-specific immunotherapy. whereas according to molecular diagnosis, prescription of three AIT vaccines would have been correct only for 5 patients (i.e., 5.3\%) (Table 1).

\section{Considerable Reduction in Disease Management Costs} through Allergen Molecule-Based Diagnosis

According to local standards the analysis of the direct and indirect costs related to diagnosis of weed, tree, and grass pollen allergy by SPT yielded EUR 23.40 per patient, whereas allergen molecule-based IgE serology with a panel of marker allergens covering the same allergen sources amounted to EUR 92.20 per patient (Table 1). Thus the costs for diagnosis obtained by SPT were EUR 68.80 lower per patient as compared to molecular IgE serology. However, SPT did not allow to discriminate between cross-sensitization and cosensitization and therefore it was not possible to identify the culprit allergen sources correctly for AIT prescription. This was only possible with purified marker allergens. It turned out that the diagnosis with marker allergens reduced the number of allergen extracts needed for AIT considerably: according to SPT results $10.5 \%$ of the patients would have received treatment with 2 extracts, and $89.5 \%$ would have been prescribed AIT with 3 extracts (Table 1). However, the more precise diagnosis with purified marker allergens showed that the majority of patients (i.e., 69.4\%) required AIT only with 1 extract, $25.3 \%$ needed 2 extracts, and only for $5.3 \%$ treatment with 3 extracts was adequate (Table 1 ). Thus, allergen moleculebased diagnosis allowed to reduce the costs for AIT from EUR 1,112.30 per patient for 3 years to EUR 521.77 (Table 1). Using allergen molecule-based diagnosis, the com- bined costs for diagnosis and 3 years of AIT for the studied group could be reduced by EUR 49,564.35 (Table 1).

\section{Discussion}

Allergic diseases are a major and continuously growing burden for the health care systems of industrialized countries [18]. Efficient diagnostic guidelines leading to appropriate treatment are therefore urgently needed. Several studies have shown that allergen molecule-based diagnosis is more precise in identifying the sensitizing allergen sources [19-23] and that it is possible to select allergens for AIT more precisely according to component-resolved diagnosis with allergen molecules than based on diagnosis with allergen extracts $[10,11]$. However, to the best of our knowledge, our study is the first to investigate in a real-life setting whether allergen molecule-based testing is more cost-effective in selecting the correct AIT vaccines than conventional allergy diagnosis based on SPT with allergen extracts. Patients in our study were tested by skin testing which likewise can be considered a provocation test taking additionally co-occurrence of symptoms with the pollen season into consideration. This is a standard procedure for diagnosing pollen allergies all over the world. The assessment of IgE binding to pollen extracts usually complements this procedure, but in most cases delivers similar results as skin testing and causes only additional costs. Therefore, serology to extracts has not been performed in our study. Furthermore, allergen extracts in (e.g., in ImmunoCAP) include cross-reactive components such as
96

Int Arch Allergy Immunol 2017;173:93-98 DOI: $10.1159 / 000477442$
Saltabayeva et al. 
profilins, polcalcins, and cross-reactive carbohydrates similar as the extracts used for SPT and one would therefore expect to find the same discrepancy as between SPT with allergen extracts and diagnosis with marker allergens.

We found that SPT with pollen allergen extracts delivered fast diagnostic information, SPT can be performed by the allergologist without the need for a laboratory, and the costs for the diagnostic reagents were on an average EUR 68.80 lower per patient than those for in vitro serology. However, allergen molecule-based IgE serology allowed not only identifying the disease-causing allergen source for each of the tested patients but was more precise in identifying the disease-causing allergen sources than allergen extract-based SPT. As a result, fewer allergen extracts were needed for AIT which led to a tremendous reduction in treatment costs in the studied group of patients. Since extract-based SPT diagnosis was ambiguous in identifying the disease causing allergen sources, more extracts were needed for treatment which amounted to EUR 1,112.30 per patient for 3 years. Allergen molecule-based testing reduced these costs to EUR 521.77, so that the total costs could be reduced by EUR 49,564.35 for the studied group of patients and for a 3-year treatment period. Our study thus shows that allergen molecule-based diagnosis for prescription of AIT allows reducing the costs for AIT treatment and the total costs for diagnosis and treatment in a substantial manner.

We think that the discrepancies between the results obtained by extract-based SPT and allergen moleculebased testing are due to sensitization to cross-reactive allergens which occur in pollen of unrelated plant species such as profilins, calcium-binding allergens, and other cross-reactive allergens [24-26]. In fact, a recent study conducted in Italy showed that $23 \%$ of pollen-sensitized patients had IgE antibodies to profilins [27] and frequent sensitization to cross-reactive pollen allergens has been reported for many countries such as the southern parts of Europe $[7,21,28,29]$. In fact, patients in Astana, Kazakhstan, are exposed simultaneously to pollen from several unrelated plant species due to a short summer period with copollination of trees, grasses, and weeds. In addition, Astana is located in a spacious steppe exposed to winds from different directions carrying a variety of pollen from different regions. Interestingly, it turned out that mugwort was the most important source for pollen sensitization in Astana, which is in agreement with pollen count data showing that the flowering period of Artemisia has strongly increased during the recent years [16]. Contaminations of allergen extracts used for skin testing with other pollens cannot be excluded and may be another possibility for over-diagnosis.

Economic Efficacy of Component-

Resoloved Diagnosis for AIT
In this context it should be mentioned that allergen extracts which are currently used for skin testing in Europe do not fulfil the standards of regulatory authorities, and it is possible that they will soon not be available anymore [30].

One limitation of our study is that it can only investigate the costs needed for correct diagnosis and the correct selection of the AIT vaccine. The responsiveness to AIT will depend on many other factors including the quality of the AIT vaccine, the representation of the individual allergens in the AIT vaccine which may reflect the sensitization profile to the individual allergens in the extract of certain patients but not of others, the immunogenicity of the allergens in the extract, and the ability of the patient to mount a protective immune response. The issue of responsiveness can only be evaluated in a large prospective, double-blind, placebo-controlled clinical trial which cannot be performed as a real-life study like our study. We are also aware that the results from our real-life economic study are applicable only for pollen AIT in Kazakhstan and cannot be generalized, but it has been shown for southern parts of Europe that allergen molecule-based testing would have changed AIT prescription also in these regions substantially $[10,11]$. So far no studies are available which have analyzed how allergen molecule-based diagnosis would affect AIT prescriptions for indoor allergen sources, but several studies have shown that molecular diagnosis is also more precise in identifying sensitizing indoor allergen sources as compared to allergen extract-based diagnosis [31-33]. Should further studies in different populations, regions, and different allergen sources show a similar cost-efficacy of molecular allergen-based diagnosis over allergen extract-based diagnosis for AIT prescription, molecular allergy diagnosis will become standard practice and help to reduce the continuously exploding health care costs for allergic diseases.

\section{Acknowledgements}

This study was performed within the framework of the International Network of Universities for Molecular Allergology and Immunology, Vienna, Austria. The study was supported in part by grant F4605 of the Austrian Science Fund (FWF).

\section{Disclosure Statement}

Rudolf Valenta has received research grants from Biomay AG, Vienna, Austria, Thermofisher, Uppsala, Sweden, and Fresenius Medical Care, Bad Homburg, Germany, and serves as a consultant for these companies. The other authors declare no financial support received from any company for this study. Felix Gastager is an employee of HVD Vertriebs-Gesellschaft m.b.H., Vienna, Austria.

Int Arch Allergy Immunol 2017;173:93-98 DOI: $10.1159 / 000477442$ 


\section{References}

1 Valenta R, Duchene M, Vrtala S, Birkner T, Ebner C, Hirschwehr R, et al: Recombinant allergens for immunoblot diagnosis of treepollen allergy. J Allergy Clin Immunol 1991; 88:889-894.

2 Valenta R, Lidholm J, Niederberger V, Hayek B, Kraft D, Grönlund H: The recombinant allergen-based concept of component-resolved diagnostics and immunotherapy (CRD and CRIT). Clin Exp Allergy 1999;29:896-904.

3 Hiller R, Laffer S, Harwanegg C, Huber M, Schmidt WM, Twardosz A, et al: Microarrayed allergen molecules: diagnostic gatekeepers for allergy treatment. FASEB J 2002; 16:414-416.

4 Ferreira F, Hawranek T, Gruber P, Wopfner N, Mari A: Allergic cross-reactivity: from gene to the clinic. Allergy 2004;59:243-267.

5 Canonica GW, Ansotegui IJ, Pawankar R, Schmid-Grendelmeier P, van Hage M, BaenaCagnani CE, et al: A WAO-ARIA-GA ${ }^{2} L E N$ consensus document on molecular-based allergy diagnostics. World Allergy Organ J 2013;6:17.

6 Kazemi-Shirazi L, Niederberger V, Linhart B, Lidholm J, Kraft D, Valenta R: Recombinant marker allergens: diagnostic gatekeepers for the treatment of allergy. Int Arch Allergy Immunol 2002;127:259-268.

7 Valenta R, Twaroch T, Swoboda I: Component-resolved diagnosis to optimize allergenspecific immunotherapy in the Mediterranean area. J Investig Allergol Clin Immunol 2007;17:36-40.

8 Birkner T, Rumpold H, Jarolim E, Ebner H, Breitenbach M, Skvaril F, et al: Evaluation of immunotherapy-induced changes in specific IgE, IgG and IgG subclasses in birch pollen allergic patients by means of immunoblotting. Correlation with clinical response. $\mathrm{Al}-$ lergy 1990;45:418-426.

9 Schmid-Grendelmeier P: Recombinant allergens routine diagnostics or still only science. Hautarzt 2010;61:946-953.

10 Sastre J, Landivar ME, Ruiz-García M, Andregnette-Rosigno MV, Mahillo I: How molecular diagnosis can change allergen-specific immunotherapy prescription in a complex pollen area. Allergy 2012;67:709-711.

11 Stringari G, Tripodi S, Caffarelli C, Dondi A, Asero R, Di Rienzo Businco A, et al: The effect of component-resolved diagnosis on specific immunotherapy prescription in children with hay fever. J Allergy Clin Immunol 2014;134: 75-81.

12 Larché M, Akdis CA, Valenta R: Immunological mechanisms of allergen-specific immunotherapy. Nat Rev Immunol 2006;6:761771.
13 Durham SR, Walker SM, Varga EM, Jacobson MR, O’Brien F, Noble W, et al: Long-term clinical efficacy of grass-pollen immunotherapy. N Engl J Med 1999;341:468-475.

14 Jacobsen L, Niggemann B, Dreborg S, Ferdousi HA, Halken S, Host A, et al: Specific immunotherapy has long-term preventive effect of seasonal and perennial asthma: 10-year follow-up on the PAT study. Allergy 2007;62: 943-948.

15 Cox L: Allergy immunotherapy in reducing healthcare cost. Curr Opin Otolaryngol Head Neck Surg 2015;23:247-254

16 Zhumambaeva S, Rosenson R; Morenko M, Shaidarov M, Zatonskikh V, Kazangapova A et al: The peculiarities of different types of chronic rhinitis in children and adolescents in Kazakhstan. Iran J Public Health 2013;42: 374-379.

17 Bousquet J, Heinzerling L, Bachert C, Papadopoulos NG, Bousquet PJ, Burney PG, et al: Practical guide to skin prick tests in allergy to aeroallergens. Allergy 2012;67:18-24.

18 Zuberbier T, Lotvall J, Simoens S, Subramanian SV, Church MK: Economic burden of inadequate management of allergic diseases in the European Union: a $\mathrm{GA}^{2} \mathrm{LEN}$ review. Allergy 2014;69:1275-1279.

19 Moverare R, Westritschnig K, Svensson M, Hayek B, Bende M, Pauli G, et al: Different IgE reactivity profiles in birch pollen-sensitive patients from six European populations revealed by recombinant allergens: an imprint of local sensitization. Int Arch Allergy Immunol 2002;128:325-335

20 Borghesan F, Bernardi D, Plebani M: In vivo and in vitro allergy diagnostics: it's time to reappraise the costs. Clin Chem Lab Med 2007; 45:391-395.

21 Douladiris N, Savvatianos S, Roumpedaki I, Skevaki C, Mitsias D, Papadopoulos NG: A molecular diagnostic algorithm to guide pollen immunotherapy in southern Europe: towards component-resolved management of allergic diseases. Int Arch Allergy Immunol 2013;162:163-172.

22 Becker S, Schlederer T, Kramer MF, Haack M, Vrtala S, Resch Y, et al: Real-life study for the diagnosis of house dust mite allergy - the value of recombinant allergen-based IgE serology. Int Arch Allergy Immunol 2016;170:132137.

23 Ma S, Nie L, Li H, Wang R, Yin J: Componentresolved diagnosis of peanut allergy and its possible origins of sensitization in China. Int Arch Allergy Immunol 2016;169:241-248.
24 Valenta R, Duchene M, Pettenburger K, Sillaber C, Valent P, Bettelheim P, et al: Identification of profilin as a novel pollen allergen; IgE autoreactivity in sensitized individuals. Science 1991;253:557-560.

25 Seiberler S, Scheiner O, Kraft D, Lonsdale D, Valenta R: Characterization of a birch pollen allergen, Bet v III, representing a novel class of $\mathrm{Ca}^{2+}$ binding proteins: specific expression in mature pollen and dependence of patients IgE binding on protein-bound $\mathrm{Ca}^{2+}$. EMBO J 1994;13:3481-3486.

26 Niederberger V, Hayek B, Vrtala S, Laffer S, Twardosz A, Vangelista L, et al: Calciumdependent immunoglobulin $\mathrm{E}$ recognition of the apo- and calcium-bound form of a cross-reactive two EF-hand Timothy grass pollen allergen, Phl p 7. FASEB J 1999;13: 843-856

27 Asero R, Tripodi S, Dondi A, Di Rienzo Businco A, Sfika I, Bianchi A, et al: Prevalence and clinical relevance of $\operatorname{IgE}$ sensitization to profilin in childhood: a multicenter study. Int Arch Allergy Immunol 2015;168:25-31.

28 Passalacqua G, Melioli G, et al: The additional values of microarray allergen assay in the management of polysensitized patients with respiratory allergy. Allergy 2013;68:10291033.

29 Sastre J, Rodríguez F, Campo P, Laffond E, Marín A, Alonso MD: Adverse reactions to immunotherapy are associated with different patterns of sensitization to grass allergens. Allergy 2015;70:598-600.

30 Klimek L, Hoffmann HJ, Renz H, Demoly P, Werfel T, Matricardi PM, Muraro A, SchmidGrendelmeier P, Cardona V, Papadopoulos NG: Diagnostic test allergens used for in vivo diagnosis of allergic diseases are at risk: a European perspective. Allergy 2015;70:13291331.

31 Grönlund H, Saarne T, Gafvelin G, van Hage M: The major cat allergen, Fel d 1, in diagnosis and therapy. Int Arch Allergy Immunol 2010;151:265-267.

32 Curin M, Swoboda I, Wollmann E, Lupinek C, Spitzauer S, van Hage M, Valenta R: Microarrayed dog, cat, and horse allergens show weak correlation between allergen-specific IgE and IgG responses. J Allergy Clin Immunol 2014;133:918-921.

33 Apostolovic D, Sánchez-Vidaurre S, Waden K, Curin M, Grundström J, Gafvelin G, et al: The cat lipocalin Fel d 7 and its cross-reactivity with the dog lipocalin Can $\mathrm{f} 1$. Allergy 2016;71:1490-1495. 